\title{
INTEGRAL observation of the accreting pulsar 1E1145.1-6141
}

\author{
C. Ferrigno ${ }^{1}$, A. Segreto ${ }^{1}$, T. Mineo ${ }^{1}$, A. Santangelo ${ }^{2}$, and R. Staubert ${ }^{2}$ \\ 1 IASF-Pa, via Ugo la Malfa 153, 90146 Palermo, Italy \\ e-mail: ferrigno@ifc.inaf.it \\ 2 IAAT, Abt. Astronomie, Universität Tübingen, Sand 1, 72076 Tübingen, Germany
}

Received 10 September 2007 / Accepted 5 December 2007

\section{ABSTRACT}

\begin{abstract}
Aims. We analyze $1050 \mathrm{ks}$ of INTEGRAL data of the high mass X-ray binary pulsar 1E 1145.1-6141 to study its properties over a long time baseline, from June 2003 to June 2004, with wide spectral coverage.

Methods. We study three high luminosity episodes, two of them at the system apoastron, three brightening with lower intensity, two at the periastron, and one extended period of intermediate luminosity spanning one orbital cycle. We perform a timing analysis to determine the pulse period and pulse profiles at different energy ranges. We also analyze the broad band phase averaged spectrum of different luminosity states and perform phase resolved spectroscopy for the first flare.

Results. From the timing analysis, we find a pulse period of $\sim 297 \mathrm{~s}$ around MJD 53000 with a significant scatter around the mean value. From the spectral analysis we find that the source emission can be described by an absorbed bremsstrahlung model in which the electron temperature varies between $\sim 25$ and $\sim 37 \mathrm{keV}$, without any correlation with luminosity, and the intrinsic absorbing column is constantly of the order of $10^{23} \mathrm{~cm}^{-2}$. Phase resolved spectral analysis reveals a different temperature of the plasma in the ascending and descending edges of the pulse during the first flare. This justifies the pulse maximum shift by $\sim 0.4$ phase units between 20 and $100 \mathrm{keV}$ observed in the pulse profiles.

Conclusions. The comparison with the previous period measurements reveals that the source is currently spinning down, in contrast to the long term secular trend observed so far, indicating that at least a temporary accretion disk is formed. The study of the spectral property variations with respect to time and spin phase suggests the presence of two emitting components at different temperatures whose relative intensity varies with time.
\end{abstract}

Key words. X-rays: binaries - stars pulsars: individual: 1E 1145.1-6141

\section{Introduction}

X-ray Binary Pulsars (XRBPs) were discovered more than 30 years ago with the pioneer observation of Cen $\mathrm{X}-3$ by Giacconi et al. (1971). Although the basic mechanisms of the pulsed emission were quickly understood (Pringle \& Rees 1972; Davidson \& Ostriker 1973), many puzzling aspects still persist. In particular, their wide band spectral behavior has not been completely explained on the basis of a self consistent physical model; spectra are successfully described by phenomenological models such as a power law modified at high-energy by an exponential cut-off and at low energy by intrinsic photo-electric absorption plus a thermal component at $\sim 0.1 \mathrm{keV}$ (Coburn et al. 2002; di Salvo et al. 2004). XRBPs are powered by the accretion of ionized gas from a nearby companion, an $\mathrm{O}$ or B star $\left(M \geq 5 M_{\odot}\right)$ in High Mass X-ray Binaries (HMXBs), a later than type A star or a white dwarf $\left(M \leq M_{\odot}\right)$ in Low Mass $\mathrm{X}$-ray Binaries (LMXBs) onto the surface of a black hole or neutron star (NS). If the compact object is highly magnetized, with a surface field as high as $10^{12} \mathrm{G}$, the plasma is threaded at the magnetospheric boundary, and then funneled to the magnetic poles (Pringle \& Rees 1972; Davidson \& Ostriker 1973) along the field lines; pulsation is generated because of the nonalignment between the magnetic and the rotational axes. In HMXBs the plasma can be captured directly from the slow and dense equatorial stellar wind of a Be star, or from the radiation driven wind of an early type super-giant. In brighter systems, accretion can be also powered by Roche-lobe overflow via an accretion disk.
The systems with Be companions (often with appreciable orbital eccentricity) are normally quiescent but sometimes they flare when the NS passes through the companion's disk. During these episodes the luminosity may increase by a large factor, a temporary accretion disk may form, and the NS generally spins up. The bright HMXBs accreting from a stable accretion disk via Roche lobe overflow have rather short spin and orbital periods, nearly circular orbits, and can exhibit torque reversal episodes superimposed on a spin-up trend as observed for Cen X-3 (Bildsten et al. 1997). Most wind-fed HMXBs with a super-giant companion have long pulse periods (hundreds of seconds), moderate and rather stable luminosity $\left(L_{\mathrm{X}} \simeq 10^{35-37} \mathrm{erg} \mathrm{s}^{-1}\right)$, and can spin-up or spin-down depending on the transfer of angular momentum from the wind to the magnetosphere. The pulse period evolution is then characterized by red-noise fluctuations due to the irregular accretion flow as in the case of Vela X-1 (Bildsten et al. 1997). Simulations have shown that a prograde or retrograde accretion disk, which is not necessarily a true viscous disk, may form and disrupt on time scales of $10^{3} \mathrm{~s}$, causing changes in the sign of the pulse period derivative (Blondin et al. 1990), as observed in the controversial case of the system with a supergiant companion OAO 1657-415 (Baykal 1997; İnam \& Baykal 2000). A temporary accretion disk is also suggested to explain the short episodes of spin-up, accompanied by luminosity enhancements, in the wind-fed binary GX 301-2 (Koh et al. 1997; İnam \& Baykal 2000).

From the Galactic plane monitoring performed with the INTEGRAL satellite, a new class of HMXBs containing a 
neutron star and super-giant (SG) donors has been identified: the Super-giant Fast X-ray Transients (SFXT), characterized by short outbursts of X-ray emission (Negueruela et al. 2006; Sguera et al. 2005, 2006). The transient behavior of these sources is explained by the motion in wide orbits (10 stellar radii) through a clumpy SG wind (in't Zand 2005; Negueruela et al. 2006), or to the recurrent passage of the NS through a denser equatorial wind component (Sidoli et al. 2007).

In this paper, we report on the INTEGRAL observations of one of the less known HMXBs located very close (17') to the extensively studied 4U 1145-61 (see e.g. Stevens et al. 1997), 1E 1145.1-6141. Optical spectroscopy of its B2 Iae super-giant companion yields a distance of $(8.5 \pm 1.5) \mathrm{kpc}$ (Densham \& Charles 1982). X-ray spectral analysis was first performed using the data in the energy range $0.5-3 \mathrm{keV}$ from the imaging proportional counter of the Einstein observatory (Lamb et al. 1980) finding a column density of $N_{\mathrm{H}}=(3 \pm 2) \times 10^{22} \mathrm{~cm}^{-2}$. This value was confirmed, using the proportional counter array of the RXTE satellite in the energy range $2-60 \mathrm{keV}$, by Ray \& Chakrabarty (2002) who modeled the spectrum using an absorbed cut-off power law with index $\Gamma=1.4$, cut-off energy $E_{\mathrm{c}}=6.4 \mathrm{keV}$ and $e$-folding energy $E_{\mathrm{f}}=18 \mathrm{keV}$. They also measured the orbital parameters of the binary system finding an eccentric $(e=0.20)$ orbit with period $P=14.365$ days, $a \sin i=99.4$ lt-sec, periastron epoch $T_{0}=51008.1 \mathrm{MJD}$, and longitude of periastron $\omega=-52^{\circ}$. They proposed, on the basis of previous observations, a secular spin-up trend of $\sim 10^{-9} \mathrm{~s} / \mathrm{s}$ between 1975 and 2000 (Ray \& Chakrabarty 2002). The NS emission was characterized by a pulsed fraction of $\sim 50 \%$ in the $4-20 \mathrm{keV}$ energy band and an Xray luminosity of $\sim 10^{36} \mathrm{erg} / \mathrm{s}$ (Hutchings et al. 1981; Grebenev et al. 1992; Ray \& Chakrabarty 2002). Such a low luminosity is inconsistent with Roche lobe overflow for this class of binaries and indicates that the NS is almost certainly accreting from the companion wind. The pulse shape has been studied using the ART-B telescope on board the GRANAT observatory in the 3-60 keV energy range by Grebenev et al. (1992) who found evidence of a notch between the maximum and the minimum of the pulse; this feature also appeared in the pulse shape obtained with RXTE (Ray \& Chakrabarty 2002).

Our paper studies the spectral and timing properties of 1E 1145.1-6141 between March 2003 and June 2004 using the data from European Space Agency's International Gamma-Ray Astronomy Laboratory (INTEGRAL) and is organized as follows: in Sect. 2 we describe the observations, in Sect. 3 we illustrate the scientific results which are discussed in Sect. 4, and in Sect. 5 we draw our conclusions.

\section{Observation and data analysis}

INTEGRAL, launched in October 2002, carries 3 co-aligned coded mask telescopes.

- IBIS (Imager on Board the INTEGRAL Satellite; Ubertini et al. 2003), which allows for 12 arcmin FWHM angular resolution imaging in the energy range from $15 \mathrm{keV}$ to $600 \mathrm{keV}$ with energy resolution of $8 \%$ at $100 \mathrm{keV}$ and $8^{\circ}$ fully coded field of view. It is composed of a low energy CdTe detector (15-600 keV), ISGRI (Lebrun et al. 2003), and a CsI layer (175 keV-10 MeV), PICsIT (Labanti et al. 2003);

- SPI (SPectrometer on INTEGRAL; Vedrenne et al. 2003), sensitive from $20 \mathrm{keV}$ to $8 \mathrm{MeV}$ with an angular resolution of $2.5^{\circ}$ and an energy resolution of a $0.2 \%$ at $1.3 \mathrm{MeV}$; the fully coded field of view is $16^{\circ}$;
- JEM-X (Joint European X-ray Monitor; Lund et al. 2003) that includes two independent units $J E M-X 1$ and $J E M-X 2$, sensitive from $3 \mathrm{keV}$ to $34 \mathrm{keV}$, with an angular resolution of $1 \mathrm{arcmin}$ and an energy resolution of $13 \%$ at $10 \mathrm{keV}$; its fully coded field of view is $4.8^{\circ}$.

INTEGRAL observations are divided in short periods (of $\sim 2000 \mathrm{~s}$ each), called science windows (SCWs), during which the telescope maintains the same pointing. From one science window to another, the pointing follows a dithering or a survey strategy as chosen in the observing program (Winkler et al. 2003).

To reduce and analyze $J E M-X$ data we used the Off-line Science Analysis (OSA) software version 5.1; for ISGRI data we exploited also the software described in Ferrigno et al. (2007) and Segreto \& Ferrigno (2007). For the spectral analysis we used the standard XSPEC package version 12.2.

In this paper we use the publicly available INTEGRAL data in which 1E 1145.1-6141 was visible before June 2004. Due to the ISGRI large field of view it has been possible to observe the source for a total of 1.08 Ms between June 2003 and June 2004, with a nearly continuous coverage of 2 binary orbits in 2004, and a sparse coverage of two and a half orbits in 2003. We limited our analysis to ISGRI and JEM-X data because the source is too weak to be studied with SPI. As summarized in Table 1, JEM$X$ detected the source only in a sub-set of the available SCWs, because of its smaller field of view with respect to ISGRI.

As can be seen from the light curves of Fig. 1, we detected three flares of about the same intensity (labeled $\mathrm{H} 1, \mathrm{H} 2$ and $\mathrm{H} 3$, where " $\mathrm{H}$ " is an abbreviation for High luminosity), the first two occurred at the NS apoastron, the last while the NS was moving away from the companion. Apoastron flares of this source have been observed by the Burst Alert Telescope on board Swift and reported by Corbet et al. (2007).

At MJD 53144 (day 1600 in the scale of Fig. 1) the source was so weak that it was pointless to perform any analysis. In the following orbital cycle, it exhibited weak and irregular luminosity increases, none of which was intense enough to allow a dedicated analysis. We then treated these data as a unique observation named EI, meaning that the source had an Extended Intermediate luminosity level. We divided the rest of the observations into three intervals labeled L1, L2 and L3 (where "L" is an abbreviation for Low luminosity), during which the source exhibited less pronounced brightening. In the interval selection we excluded the neighboring SCWs with few source counts to improve the $\mathrm{S} / \mathrm{N}$ and we separated L3 from EI since it has the appearance of a low luminosity flare occurring near the system periastron, such as L1, rather than an extension of EI. The L2 episode occurred while the NS was approaching the companion.

The H3 episode was reported by Bodaghee et al. (2004), who stated that a flare of this intensity has never been mentioned in the literature for 1E 1145.1-6141. But it should be noted that the source did present brightening of the same intensity during the episodes here called $\mathrm{H} 1$ and $\mathrm{H} 2$.

To perform the timing analysis, we transformed the event arrival times for each time interval listed in Table 1 to the Solar System Barycenter and then to the Binary System Barycenter using the orbital parameters found by Ray \& Chakrabarty (2002). We determined the pulse period with a version of the phaseshift method described extensively in Ferrigno et al. (2007) and briefly summarized here. Using the ISGRI data, which guarantee the best signal to noise ratio $(\mathrm{S} / \mathrm{N})$ and time coverage, we find a tentative pulse period from the Fourier analysis of the light curve. Then, using this value, we accumulate a pulse profile in 


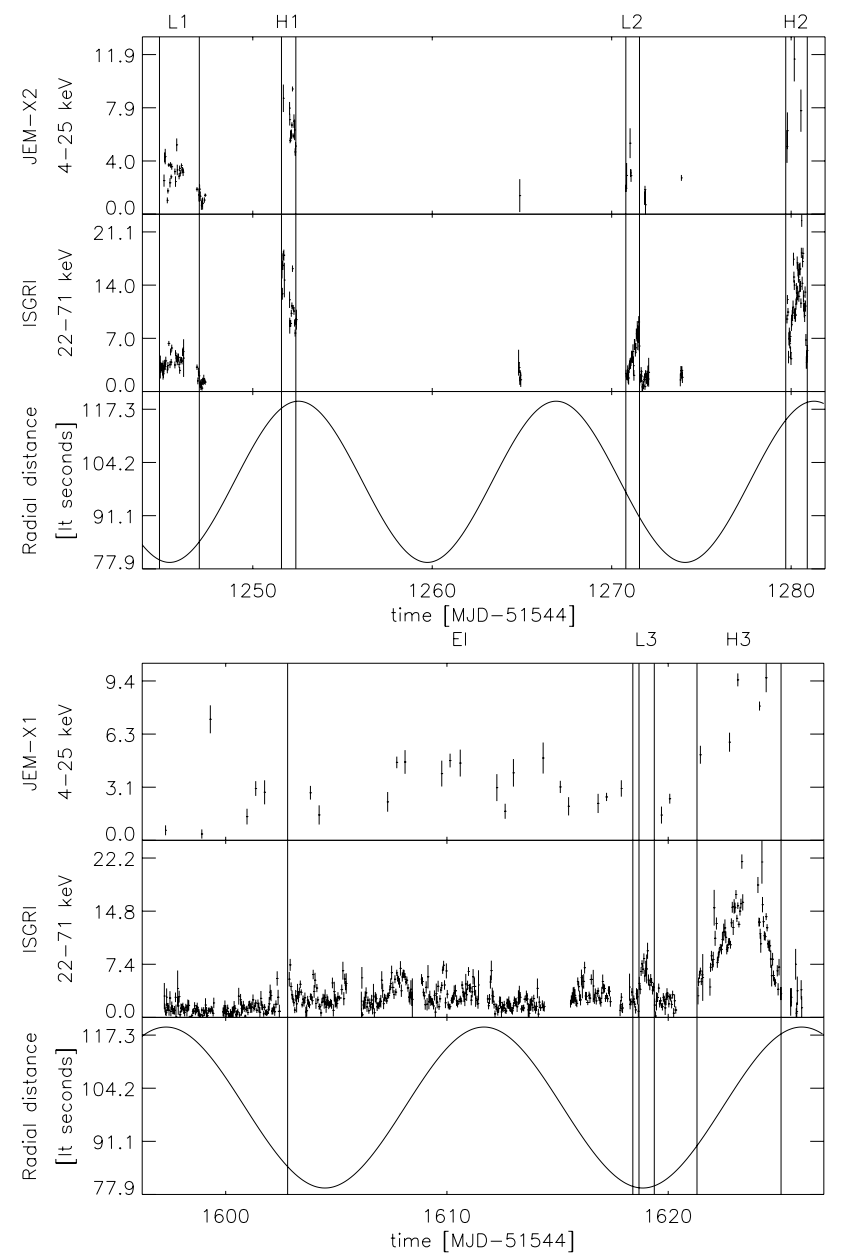

Fig. 1. Light curve of 1E 1145-6141 during the INTEGRAL observations. From top to bottom, the data of JEM-X (4-25 keV) and ISGRI (22-71 keV) (from http://isdc.unige.ch/?Data+sources) and the distance between the stars of the binary system expressed in light seconds as computed from the ephemeris measured by Ray \& Chakrabarty (2002). The time intervals in which we performed the analysis are indicated in the figure between vertical solid lines and labeled with the corresponding name.

each science window and determine the phase of the pulse maximum. Finally, from a parabolic fit of the pulse maximum phase as a function of time, we find the optimal pulse period and an upper limit on its first derivative.

Unless differently stated, the errors in the paper are quoted at a $1 \sigma$ confidence level.

\section{Results}

\subsection{Timing analysis}

We measure the pulse period in the time intervals reported in Table 2; during L1, L2 and L3 the statistics is not sufficient for an independent determination. The pulse period derivative is always too small to be directly measured. In particular, during EI we find the $2 \sigma$ upper limit $|\dot{P}|<4 \times 10^{-9} \mathrm{~s} / \mathrm{s}$. Thus, during this orbital cycle the period variations do not exceed the long term trend.

We plot in Fig. 2 our results together with the period and the spin-up trend reported by Ray \& Chakrabarty (2002). We note that the INTEGRAL values are significantly higher than the extrapolation of the secular evolution measured by
Ray \& Chakrabarty (2002). To verify the occurrence of a torque reversal, we computed the average value of our period determinations $(296.695 \pm 0.002 \mathrm{~s})$ and of the last four $R X T E$ results ( $296.572 \pm 0.001 \mathrm{~s})$ : the difference of $0.123 \pm 0.003 \mathrm{~s}$ in five years suggests an average spin-down of $\sim 7 \times 10^{-10} \mathrm{~s} / \mathrm{s}$ in contrast to the previous spin-up trend of $-1 \times 10^{-9} \mathrm{~s} / \mathrm{s}$. The source then began to reduce its average angular speed on a time scale of years, even though fluctuations in the trend are present. At MJD 52824.0 (H2) we found a period of (296.62 \pm 0.02$)$ s, compatible with the RXTE determinations, but at more than $3 \sigma$ from the other values we measured. If the trend proposed by Ray \& Chakrabarty (2002) is correct a torque reversal would have taken place between RXTE and INTEGRAL observations around MJD 52000 ; but we cannot exclude that the source was spinning down before MJD 45 000, spinning up until MJD 50500 and then spinning up again. Unfortunately, the poor determination of the pulse period by EINSTEIN at MJD 44000 does not allow to verify this second hypothesis.

Using the values of Table 2, we produced the pulse profiles of the ISGRI data in the energy bands 15-35, 35-60, and 60$110 \mathrm{keV}$ arbitrarily setting the zero phase at the pulse minimum in the lowest energy range. The pulse profiles (Fig. 3) show a similar shape over all the observing periods. They are dominated by a single broad peak which moves to earlier phases with increasing energy, and present also a hint of a second peak at phase $\sim 0.7$, which is reminiscent of the notch between the pulse maximum and minimum reported by Grebenev et al. (1992) and Ray \& Chakrabarty (2002). However, some differences arise at higher energy where the main peak has an increasing sharpness, if the time intervals are put in the sequence EI, H3, H1, and H2.

To investigate the evolution of the pulse profile with energy, we focus on the $\mathrm{H} 1$ time interval when JEM-X exposure is long enough to accumulate pulse profiles with an acceptable $\mathrm{S} / \mathrm{N}$ below $20 \mathrm{keV}$. The pulse profiles are modeled with a Gaussian plus a constant (reduced $\chi^{2}$ between 0.8 and 1.4 for 35 degrees of freedom) as shown in Fig. 4. We find that the width of the Gaussian remains constant with an average value of $0.21 \pm 0.05$ while its centroid shifts to earlier phases for increasing energy: between 2 and $8 \mathrm{keV}$ the pulse profile peaks at pulse phase $0.5 \pm 0.2$, between 20 and $30 \mathrm{keV}$ at pulse phase $0.4 \pm 0.1$, and above $80 \mathrm{keV}$ at pulse phase $0.2 \pm 0.2$. The linear fit of the phase of the centroid position $x$ as a function of energy $E$ gives:

$x=(0.55 \pm 0.10)-(4.4 \pm 2.4) \times 10^{-3} \frac{E}{[\mathrm{keV}]}$.

Repeating the same procedure for the intervals H2, EI and H3 for the ISGRI data alone, due to the poor quality or lack of $J E M-X$ data, we find that the relation of Eq. (1) is always verified. However, the limited energy range prevents an independent determination of the centroid evolution with energy.

\subsection{Spectral analysis}

In each observing period we perform broad band phase averaged spectral analysis using ISGRI data and, whenever available, JEM-X data. We investigate several spectral models and eventually find satisfactory fits for a cut-off power law $\left(E^{-\gamma} \exp \left[-E / E_{\mathrm{f}}\right]\right)$ plus photoelectric absorption, implemented as PHABS in XSPEC using the cross sections by BalucinskaChurch \& McCammon (1992), and bremsstrahlung also attenuated by absorption. But a discrimination between these models on a statistical base is not possible because all fits give acceptable $\chi^{2}$ values (see Table 3 ). 
Table 1. Summary of the INTEGRAL observations of 1E 1145.1-6141.

\begin{tabular}{lccccccc}
\hline \hline Name & Start [MJD] & End [MJD] & \multicolumn{2}{c}{ Exposure [ks] } & \multicolumn{2}{c}{ Mean Count Rate [cts/s] } & $L(5-50 \mathrm{keV})^{*}$ \\
& & & ISGRI & JEM-X & ISGRI & JEM-X & {$\left[\mathrm{erg} \mathrm{s}^{-1}\right]$} \\
\hline L1 & 52788.8947 & 52791.0241 & 64.1 & 10.9 & $5.9 \pm 0.1$ & $3.2 \pm 0.1$ & $4.1 \times 10^{35}$ \\
H1 & 52795.6284 & 52796.4538 & 29.2 & 13.7 & $14.4 \pm 0.2$ & $6.2 \pm 0.1$ & $1.1 \times 10^{36}$ \\
L2 & 52814.7897 & 52815.5495 & 49.6 & 15.5 & $5.8 \pm 0.2$ & $4.3 \pm 0.5$ & $2.5 \times 10^{35}$ \\
H2 & 52823.7611 & 52824.9043 & 39.0 & & $14.7 \pm 0.2$ & & \\
EI & 53146.8561 & 53162.3828 & 703.5 & & $3.53 \pm 0.05$ & & \\
L3 & 53162.6755 & 53163.3679 & 42.1 & & $7.9 \pm 0.2$ & & \\
H3 & 53165.3538 & 53169.1147 & 153.4 & 2.2 & $12.2 \pm 0.1$ & $8.35 \pm 0.03$ & $1.0 \times 10^{36}$ \\
\hline
\end{tabular}

${ }^{*}$ Assuming a distance of $8.5 \mathrm{kpc}$.

Table 2. Pulse periods $(P)$ and the upper limits on its first derivatives $(\dot{P})$.

\begin{tabular}{llcc}
\hline \hline name & Reference time* $^{*}[\mathrm{MJD}]$ & $P[\mathrm{~s}]$ & $|\dot{P}|^{* *}[\mathrm{~s} / \mathrm{s}]$ \\
\hline H1 & 52796.00566 & $296.78 \pm 0.04$ & $<7 \times 10^{-6}$ \\
H2 & 52824.25522 & $296.62 \pm 0.02$ & $<6.5 \times 10^{-7}$ \\
EI & 53154.80607 & $296.698 \pm 0.003$ & $<4 \times 10^{-9}$ \\
H3 & 53167.38765 & $296.69 \pm 0.01$ & $<1.8 \times 10^{-7}$ \\
\hline
\end{tabular}

* At the reference time the pulse profile has a minimum in the 15 $35 \mathrm{keV}$ energy range.

** Upper limits are given at the $2 \sigma$ confidence level.

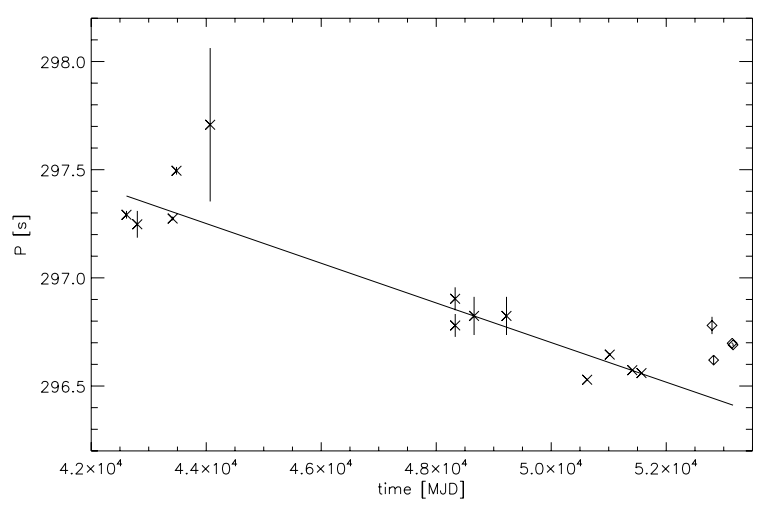

Fig. 2. Long-term evolution of the pulse period of 1E 1145.1-6141. The diamonds after MJD 52000 represent the results of our analysis. The crosses are the previous determinations, and the solid line is the spin-up trend derived by Ray \& Chakrabarty (2002).

The cut-off power law model gives best fit values with large uncertainties in most of the observing periods, probably due to a strong correlation between the spectral index and the other parameters. It is then difficult to find any significant trend of the parameters with luminosity or time; nonetheless some conclusions can be drawn: the lowest value of $N_{\mathrm{H}}\left(7.5 \pm 2.5 \times 10^{22} \mathrm{~cm}^{-2}\right)$ is significantly higher than both the Galactic column $\left(N_{\mathrm{H}}=\right.$ $1.4 \times 10^{22} \mathrm{~cm}^{-2}$; Dickey \& Lockman 1990) and the intrinsic column measured by EINSTEIN $\left((3 \pm 2) \times 10^{22} \mathrm{~cm}^{-2}\right.$, Lamb et al. 1980); the measured spectral index of the power law (1-1.5) is typical of several HMXBs (Coburn et al. 2002).

In Fig. 5, we show the spectra in the low and high luminosity states L1 and H1 together with the high energy cut-off model used by Ray \& Chakrabarty (2002) $\left(E^{-\gamma} \exp \left[-\left(E-E_{\mathrm{c}}\right) / E_{\mathrm{f}}\right]\right.$ for $E>E_{\mathrm{c}}$ and $E^{-\gamma}$ for $\left.E<E_{\mathrm{c}}\right)$. We note that in our data the

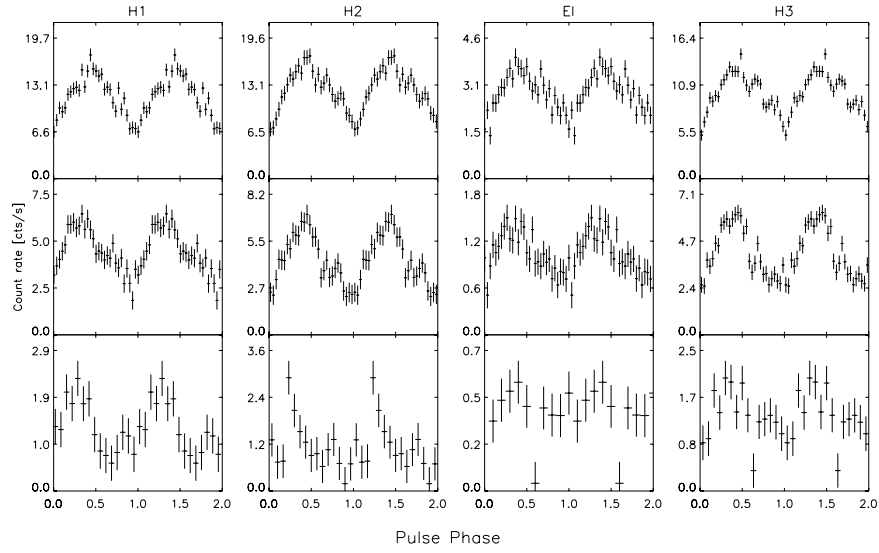

Fig. 3. Background subtracted pulse profiles of 1E 1145.1-6141 for different periods in three energy bands.

source emission is considerably harder, as confirmed by the higher value of the folding energy $E_{\mathrm{f}}=28_{-3}^{+5} \mathrm{keV}$ when the spectra during $\mathrm{H} 1$ and $\mathrm{H} 3$ are described by the same model of Ray $\&$ Chakrabarty (2002). Fitting the spectra of the other observing periods with this function, we note unreasonably large uncertainties in the parameters together with a $\chi^{2}$ not better than the values of Table 3 . It is then evident that the relatively low $\mathrm{S} / \mathrm{N}$ of the INTEGRAL observations of this source prevents the use of this phenomenological model, which has a redundant number of free parameters. Since the source brightness in our observations is not greater than in the past, we can exclude that this evolution is luminosity related.

From the best fit values of the bremsstrahlung model plotted in Fig. 6, we note that the plasma temperature is variable even if not correlated with the source luminosity. Instead, $N_{\mathrm{H}}$ is always higher than the Galactic column and does not present any significant trend. We also verify that fixing the absorbing column at its average value, the determinations of the plasma temperature do not vary significantly.

\subsection{Phase resolved spectral analysis}

During the time interval $\mathrm{H} 1$ the statistics allows us to perform broad band phase resolved spectroscopy combining data from both ISGRI and JEM-X. In the other time intervals the source is either too weak or too off-axis for $J E M-X$ to collect enough counts, and then to significantly constrain the continuum parameters.

In this analysis we adopt the absorbed bremsstrahlung model, since it gives a good description of the source emission with the lowest number of parameters. We divide the pulse in 
Table 3. Summary of the results of spectral fits with two models: cut-off power law and thermal bremsstrahlung, both modified by photoelectric absorption. The fluxes are computed directly from the source counts and are thus independent of the adopted absorption; for comparison the flux of the Crab in the $20-50 \mathrm{keV}$ energy band is $10^{-8} \mathrm{erg} \mathrm{s}^{-1} \mathrm{~cm}^{-2}$. The errors are at a $90 \%$ confidence level for one parameter of interest $\left(\chi_{\mathrm{min}}^{2}+2.7\right)$.

\begin{tabular}{lccccccc}
\hline \hline parameter & L1 & H1 & L2 & H2 & EI & L3 & H3 \\
\hline Cut-off power law & & & & & & & \\
$N_{\mathrm{H}}\left[10^{22} \mathrm{~cm}^{-2}\right]$ & $20 \pm 8$ & $7.0 \pm 2.5$ & $19_{-11}^{+15}$ & $N / A$ & $N / A$ & $N / A$ & $12 \pm 7$ \\
Photon index & $1.5 \pm 0.4$ & $1.1 \pm 0.1$ & $1.5 \pm 0.5$ & $1.4 \pm 0.2$ & $1.8 \pm 0.3$ & $1.1 \pm 0.8$ & $1.2 \pm 0.2$ \\
$E_{\mathrm{f}}[\mathrm{keV}]$ & $31_{-8}^{+16}$ & $25.5 \pm 2.5$ & $39_{-13}^{+50}$ & $28_{-5}^{+7}$ & $35_{-10}^{+18}$ & $25_{-9}^{+31}$ & $29_{-4}^{+5}$ \\
$\chi^{2} /$ d.o.f. & $218 / 197$ & $232 / 220$ & $240 / 205$ & $36 / 43$ & $64 / 51$ & $74 / 65$ & $197 / 195$ \\
\hline Bremsstrahlung & & & & & & & \\
$N_{\mathrm{H}}\left[10^{22} \mathrm{~cm}^{-2}\right]$ & $15 \pm 4$ & $10.4 \pm 1.5$ & $15 \pm 9$ & $N / A$ & $N / A$ & $N / A$ & $13 \pm 5$ \\
$k T[\mathrm{keV}]$ & $28 \pm 2$ & $33.3 \pm 1.5$ & $37 \pm 4$ & $28.5 \pm 1.0$ & $25.1 \pm 1.3$ & $35 \pm 4$ & $32 \pm 1$ \\
$\chi^{2} /$ d.o.f. & $220 / 198$ & $240 / 221$ & $240 / 206$ & $36 / 44$ & $68 / 52$ & $62 / 44$ & $198 / 196$ \\
\hline flux $\left(5-15 \mathrm{keV}^{*}\right.$ & $2.9 \pm 0.2$ & $7.47 \pm 0.15$ & $2.3 \pm 0.5$ & $N / A$ & $N / A$ & $N / A$ & $6.6 \pm 0.3$ \\
flux $(20-50 \mathrm{keV})^{*}$ & $2.9 \pm 0.1$ & $8.2 \pm 0.1$ & $2.9 \pm 0.1$ & $8.8 \pm 0.1$ & $1.96 \pm 0.04$ & $4.0 \pm 0.2$ & $7.29 \pm 0.07$ \\
\hline
\end{tabular}

${ }^{*}$ In unity of $10^{-10} \mathrm{erg} \mathrm{s}^{-1} \mathrm{~cm}^{-2}$.

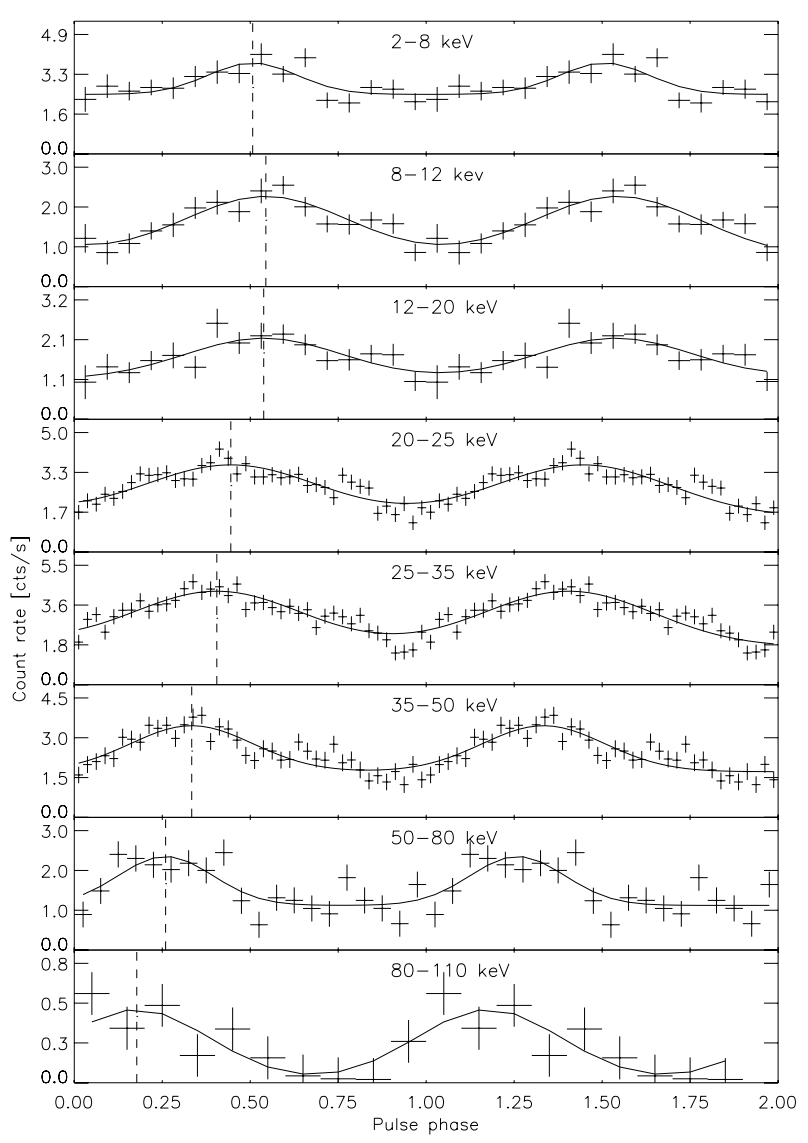

Fig. 4. Background subtracted pulse profiles of 1E 1145.1-6141 during H1. Below $20 \mathrm{keV}$ the data are from JEM-X, above from ISGRI. In each panel, the solid line is the best fit pulse profile obtained adding a Gaussian to a constant; the vertical dashed line is the position of the Gaussian centroid.

five equally spaced phase intervals and fit the spectra obtaining a reduced $\chi^{2} \sim 1$ for $\sim 100$ d.o.f. and the the best fit parameters plotted in Fig. 7. The value of $N_{\mathrm{H}}$ is approximately constant $\left(\sim 10^{23} \mathrm{~cm}^{-2}\right)$, while the plasma temperature is higher at pulse phase $0-0.4$ (ascending edge of the pulse) than at pulse phase 0.4-1 (descending edge). Averaging the fitted values, we find $k T_{0-0.4}=(40 \pm 2) \mathrm{keV}$ and $k T_{0.4-1}=(29 \pm 1) \mathrm{keV}$ corresponding to a variation of the temperature with a significance of $5 \sigma$.

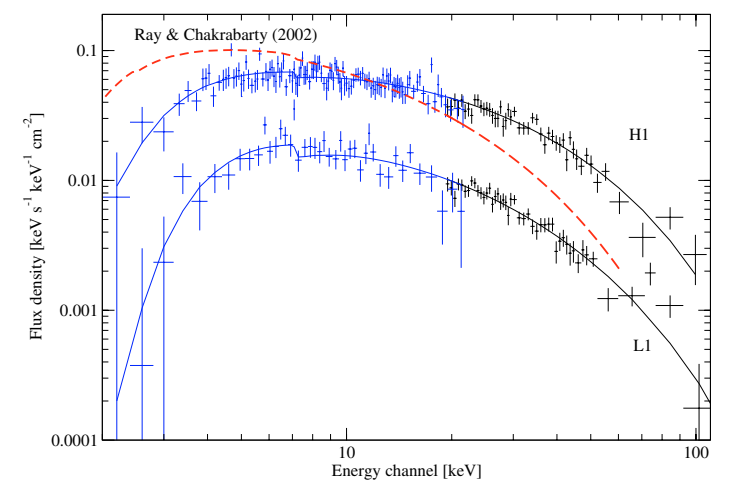

Fig. 5. Broad band unfolded energy spectra for the high state H1 and the low state $\mathrm{L} 1$. Below $\sim 20 \mathrm{keV}$ data are from $J E M-X$, above this value from ISGRI. The model in both cases is absorbed bremsstrahlung. The dashed line is the model used by Ray \& Chakrabarty (2002) with arbitrary normalization.

Fixing the value of $N_{\mathrm{H}}$ at $10^{23} \mathrm{~cm}^{-2}$ (the average over the spin period) we obtain temperature determinations compatible with the previous case, and a slight improvement in the significance of the variation $(5.3 \sigma)$. The higher temperature in the rising part of the main peak explains its shift with energy since at increasing energy the hotter emission is prominent.

The phase resolved spectral analysis in the other time intervals does not give a statistically significant variation of the plasma temperature.

\section{Discussion}

We obtain a good description of the source spectral energy distribution using the absorbed bremsstrahlung model; this does not mean, alone, that this is the real emission mechanism. To check consistency, we compute from the expression of the thermal bremsstrahlung emissivity, equation (87) of Longair (1999), the quantity $n_{\mathrm{e}}^{2} V$, where $n_{\mathrm{e}}$ is the electron density and $V$ is the volume of the emitting region for a pure hydrogen plasma emitting at the observed temperature with a power compatible to the luminosity of the source. Assuming for the accretion column a radius of $100 \mathrm{~m}$ and deriving the mass accretion rate from the $\mathrm{X}$-ray luminosity, it is possible to estimate the order of magnitude of the electron density which implies a Compton optical depth of order unity both in the vertical and in the horizontal directions of the accretion column. Therefore, it is not possible to 


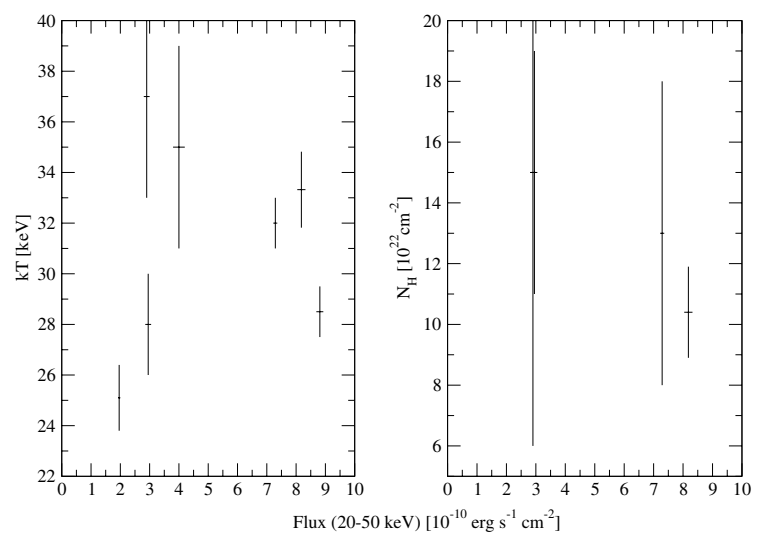

Fig. 6. Best fit parameters of the absorbed bremsstrahlung model as function of the source luminosity. In the left panel we plot the plasma temperature and in the right panel the $N_{\mathrm{H}}$ of the intrinsic photoelectric absorber. Errors are at $90 \%$ c.l. for one parameter of interest.

ensure that Compton scattering is negligible, but, on the other hand, it is not clear how much it distorts the underlying spectral energy distribution.

Becker \& Wolff (2007) recently showed that the observed spectrum of XRBPs is well described by thermal and bulk Comptonization of the optically thin bremsstrahlung and cyclotron emission from the accretion column. Using the measured luminosity and distance of the source, we tested their model on the spectrum with the best $\mathrm{S} / \mathrm{N}$, i.e. during $\mathrm{H} 1$. The fit was very good, suggesting that all the emission comes from re-processing of an underlying bremsstrahlung with an electron temperature of $\sim 13 \mathrm{keV}$ without any need for intrinsic absorption. Unfortunately, it was not possible to constrain all the model parameters, especially the magnetic field, due to the absence of absorption features. However, we note that the spectral energy distribution is very similar to absorbed bremsstrahlung and then, in data with relatively low $\mathrm{S} / \mathrm{N}$, the two models are statistically equivalent.

The values we find for the plasma temperature (higher than for the Becker and Wolff model) and for the absorbing column depth should then be regarded as a useful parametrization of the spectral energy distribution, rather than a measurement of the plasma characteristics. The strong absorption could be due to an emission turn-off at low energy, but could also be partially explained by the presence of optically thick gas in the stellar wind e.g. a bow shock formed by the X-ray pulsar radiation (Blondin et al. 1990). Unfortunately, only observations with a better spectral resolutions below $\sim 10 \mathrm{keV}$ would detect the absorption edges and then constrain significantly the column value.

More insight into the structure of the emission region can be achieved from the pulse phase resolved spectroscopy. The constant $N_{\mathrm{H}}$ at any phase indicates that the low energy part of the spectrum is governed by a process isotropically distributed around the NS or located far from it, while the bimodal temperature distribution and the presence of a notch in some pulse profiles (Fig. 3) suggest the presence of two components at different temperatures in the magnetosphere of 1E 1145.1-6141. If their relative intensity is variable with time, the different values of the plasma temperature in the phase averaged spectra between 25 and $40 \mathrm{keV}$ and the time modulation of the pulse shape would be justified. Indeed, all the analyzed spectra can be fitted with a linear combination of two bremsstrahlung models with fixed temperatures of 19 and $40 \mathrm{keV}$.
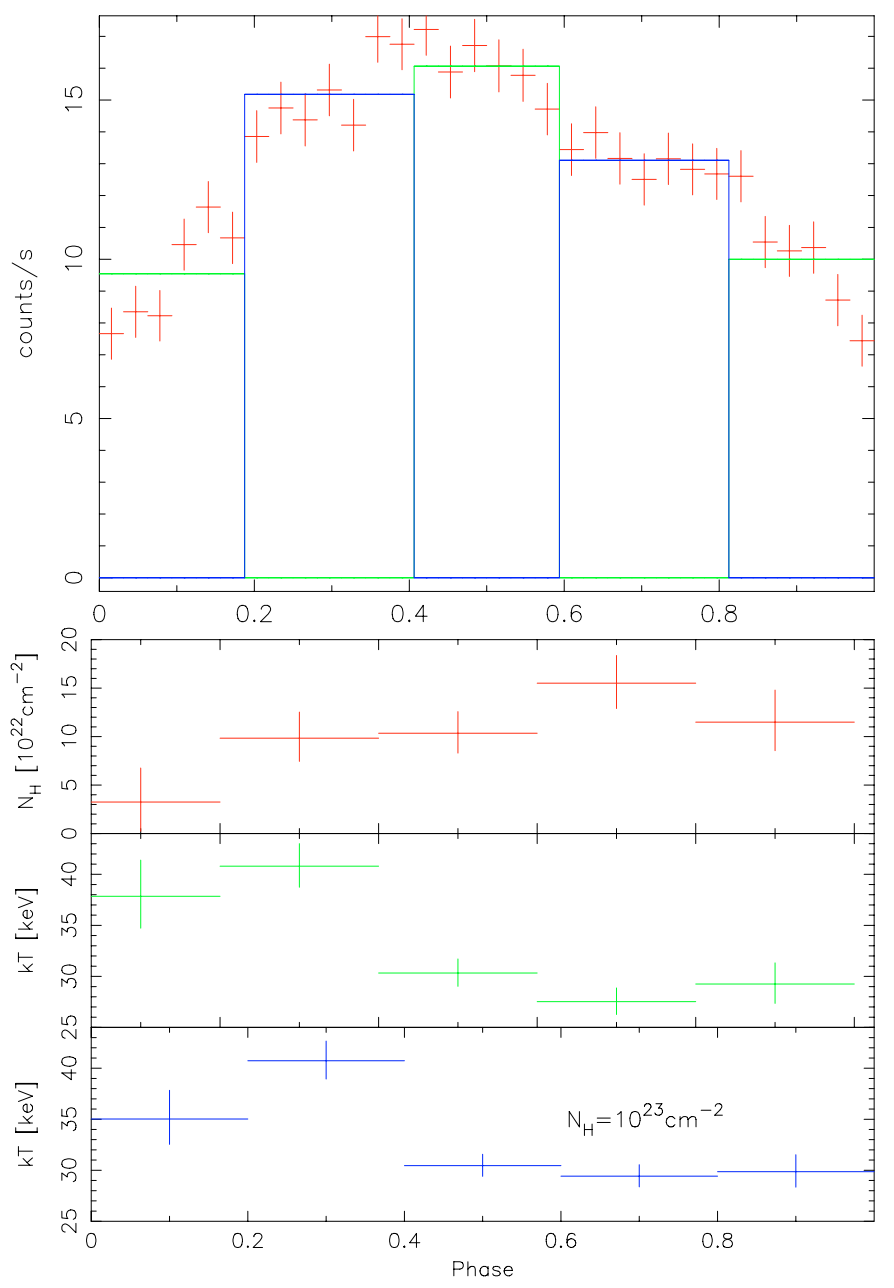

Fig. 7. Phase resolved spectral analysis results. Upper panel: the pulse profile during $\mathrm{H} 1$ in the $20-60 \mathrm{keV}$ energy range with the adopted phase intervals. Lower panel: the best fit parameters of the adopted model (photo-electric absorbed bremsstrahlung) as a function of the pulse phase during H1. From top to bottom we plot the hydrogen column density $N_{\mathrm{H}}$, the plasma temperature $k T$, where $N_{\mathrm{H}}$ is a free parameter and where $N_{\mathrm{H}}$ is held fixed at $10^{23} \mathrm{~cm}^{-2}$.

During three flares, the source reached $80-90 \mathrm{mCrab}$ in the 20-50 keV energy band. During three other periods it reached $30-40 \mathrm{mCrab}$, but for one binary orbit (EI) it presented only irregular and weak luminosity variation with an average flux of $20 \mathrm{mCrab}$. Two of the most intense flares occurred at the apoastron, the other at about two thirds of the maximum distance. At the periastron the NS reached at most one third of the maximum luminosity.

This behavior rules out the hypothesis of a spherically symmetric wind, and of a combination of disk and wind proposed to explain the periodic flares in IGR J11215-5952 (Sidoli et al. 2007) or GX 301-2 (Pravdo \& Ghosh 2001). A complex wind environment with clumps or filaments of higher density plasma is a more natural explanation of its irregular luminosity increases, but our measurements do not allow a good spectral constraint of the absorbing column, thus this hypothesis cannot be tested spectroscopically.

A clumpy stellar wind is invoked to explain the light curves of the super-giant fast X-ray transients (in't Zand 2005; Leyder et al. 2007) characterized by short irregular flares lasting from a few ks to a fraction of a day. The NS in these systems is thought to orbit at $\sim 10$ stellar radii while in persistent systems the 
separation is supposed to be smaller (Walter \& Zurita Heras 2007). We can estimate for $1 \mathrm{E} 1145.1-6141$, on the basis of the spectral type, a mass of $\sim 10 M_{\odot}$ and a stellar radius of $\sim 5 R_{\odot}$ (Habets \& Heintze 1981). From the measured orbital parameters, we derive that the NS lies at about 10 stellar radii from the companion, with a distance modulation of $20 \%$ due to the orbit eccentricity.

Although it is a persistent system, it has some characteristics similar to the SFXT: in particular the component separation and the flaring activity. However, compared to these objects, the flares are longer (from a significant fraction of a day to about four days) and have a smaller relative (4-5) intensity with respect to the persistent flux level, which, in turn, is two orders of magnitude larger. Supposing that the mechanism to capture the plasma has similar efficiency in all systems, we infer that the wind is on average denser, while the clumps are less compact than in SFXT, making the system an intermediate case between transient and persistent wind-fed HMXBs with a supergiant companion.

Unfortunately, in our case, an estimate of the mass spectrum of the clumps from the flare duration is not straightforward since the torque history is characterized by local variations, and then the formation of a temporary accretion disk is probable, which prevents the plasma from directly falling onto the NS surface. Wind clumpyness appears a common feature in binary early type stellar wind suggesting further investigations to clarify whether this can be due to the interaction with the NS or is an intrinsic characteristic of the wind.

\section{Conclusions}

We confirm that $1 \mathrm{E} 1145.1-6141$ is a persistent low luminosity binary system but we find interesting peculiarities not previously noticed.

The source was always detectable, when in the instrument field of view, and showed a complex light curve that strongly suggests a clumpy stellar wind.

The timing analysis shows that the pulse period of the source is not constant (at the $98 \%$ confidence level). These variations of the torque are expected in wind-fed systems. In addition we find that the source reversed its long term torque. The average of the measured pulse periods is higher than the average of the RXTE observation (June 1997-January 2000) while Ray \& Chakrabarty (2002) found a previous spin-up trend. Unfortunately, the lack of continuous monitoring prevents us from studing in more detail the torque history and the accretion model.

The absorbed bremsstrahlung model allows an accurate parametrization of the source spectral characteristics, even though it cannot be regarded as a coherent physical model, since the Compton optical depth of the accretion column is of the order of unity. We find that the plasma temperature varies with no correlation to the source luminosity while the absorbing column remains approximately constant. A comparison with the model adopted in the previous RXTE observations (Ray \& Chakrabarty 2002) indicates a significant hardening of the spectrum which cannot be explained by a different luminosity level.

Phase resolved spectroscopy shows that the absorbing column depth is approximately constant, and the plasma temperature in the first half of the pulse profile is $(11 \pm 2) \mathrm{keV}$ higher than in the second half. This justifies the pulse maximum shift by $\sim 0.4$ phase units between 20 and $100 \mathrm{keV}$. To describe the emission of 1E 1145.1-6141, we then propose the presence of two spectral components at different temperatures and, possibly, a layer of absorbing material isotropically surrounding the NS or located far from it. The variation of the components' relative intensity with time would also explain the oscillations of the phase averaged temperature that we observe.

Acknowledgements. The observational data used in this communication were collected by INTEGRAL, an ESA science mission for X-ray and Gamma-ray astronomy. The work was supported by the Italian Space Agency (ASI) under contract No. I/R/023/05 and by the German Space Agency (DLR) under contract nos. 50 OG 9601 and 50 OG 0501. C.F. would like to thank Rosario Iaria and the anonymous referee for their useful comments and suggestions.

\section{References}

Balucinska-Church, M., \& McCammon, D. 1992, ApJ, 400, 699

Baykal, A. 1997, A\&A, 319, 515

Becker, P. A., \& Wolff, M. T. 2007, ApJ, 654, 435

Bildsten, L., Chakrabarty, D., Chiu, J., et al. 1997, ApJS, 113, 367

Blondin, J. M., Kallman, T. R., Fryxell, B. A., \& Taam, R. E. 1990, ApJ, 356, 591

Bodaghee, A., Mowlavi, N., \& Ballet, J. 2004, The Astronomer's Telegram, 290, 1

Coburn, W., Heindl, W. A., Rothschild, R. E., et al. 2002, ApJ, 580, 394

Corbet, R., Markwardt, C., Barbier, L., et al. 2007, [arXiv: astro-ph/0703274]

Davidson, K., \& Ostriker, J. P. 1973, ApJ, 179, 585

Densham, R. H., \& Charles, P. A. 1982, MNRAS, 201, 171

di Salvo, T., Santangelo, A., \& Segreto, A. 2004, Nuclear Physics B Proc. Suppl. 132,446

Dickey, J. M., \& Lockman, F. J. 1990, ARA\&A, 28, 215

Ferrigno, C., Segreto, A., Santangelo, A., et al. 2007, A\&A, 462, 995

Giacconi, R., Gursky, H., Kellogg, E., Schreier, E., \& Tananbaum, H. 1971, ApJ, 167, L67

Grebenev, S. A., Pavlinsk, M. N., \& Syunyaev, R. A. 1992, Sv A Lett. 18, 228

Habets, G. M. H. J., \& Heintze, J. R. W. 1981, A\&AS, 46, 193

Hutchings, J. B., Crampton, D., \& Cowley, A. P. 1981, AJ, 86, 871

İnam, S. Ç., \& Baykal, A. 2000, A\&A, 353, 617

in't Zand, J. J. M. 2005, A\&A, 441, L1

Koh, D. T., Bildsten, L., Chakrabarty, D., et al. 1997, ApJ, 479, 933

Labanti, C., Di Cocco, G., Ferro, G., et al. 2003, A\&A, 411, L149

Lamb, R. C., Markert, T. H., Hartman, R. C., Thompson, D. J., \& Bignami, G. F. 1980, ApJ, 239, 651

Lebrun, F., Leray, J. P., Lavocat, P., et al. 2003, A\&A, 411, L141

Leyder, J.-C., Walter, R., Lazos, M., Masetti, N., \& Produit, N. 2007, A\&A, 465, L35

Longair, M. S. 1999, in X-Ray Spectroscopy in Astrophysics, ed. J. van Paradijs, \& J. A. M. Bleeker, LNP, 520, p24

Lund, N., Budtz-Jørgensen, C., Westergaard, N. J., et al. 2003, A\&A, 411, L231

Negueruela, I., Smith, D. M., Reig, P., Chaty, S., \& Torrejón, J. M. 2006, in The

X-ray Universe 2005, ed. A. Wilson, ESA Special Publication, 604, 165-170

Pravdo, S. H., \& Ghosh, P. 2001, ApJ, 554, 383

Pringle, J. E., \& Rees, M. J. 1972, A\&A, 21, 1

Ray, P. S., \& Chakrabarty, D. 2002, ApJ, 581, 1293

Segreto, A., \& Ferrigno, C. 2007, [arXiv:0709.4132]

Sguera, V., Barlow, E. J., Bird, A. J., et al. 2005, A\&A, 444, 221

Sguera, V., Bazzano, A., Bird, A. J., et al. 2006, ApJ, 646, 452

Sidoli, L., Romano, P., Mereghetti, S., et al. 2007,

[arXiv: astro-ph/0710.1175]

Stevens, J. B., Reig, P., Coe, M. J., et al. 1997, MNRAS, 288, 988

Ubertini, P., Lebrun, F., Di Cocco, G., et al. 2003, A\&A, 411, L131

Vedrenne, G., Roques, J.-P., Schönfelder, V., et al. 2003, A\&A, 411, L63

Walter, R., \& Zurita Heras, J. 2007, A\&A, 476, 335

Winkler, C., Courvoisier, T. J.-L., Di Cocco, G., et al. 2003, A\&A, 411, L1 